\title{
IT Adoption, Diffusion, and Evaluation in Healthcare Minitrack Introduction
}

\author{
Leigh Cellucci \\ East Carolina University \\ celluccie@ecu.edu
}

\author{
Arnold Kamis \\ Suffolk University \\ akamis@suffolk.edu
}

\author{
Ton Spil \\ University of Twente \\ a.a.m.spill@utwente.nl
}

\author{
Karoly Bozan \\ Duquesne University \\ bozank@duq.edu
}

The adoption, diffusion, and evaluation of IT in healthcare continue to present challenges to organizations and society, as well as to researchers. IT is seen as an enabler of change both nationally and locally in healthcare organizations. However, IT adoption decisions in healthcare are complex because of the uncertainty of benefits and the rate of change of technology.

The papers in this minitrack utilize numerous research approaches. Delphi studies, surveys, interviews, and longitudinal case studies all provide different methods to investigate the range of issues underlying the successful adoption, implementation, and evaluation of IT. These approaches are all represented in this minitrack.

The first paper investigates the role of user characteristics and patterns of EHR use. Using theory of affordances, it conceptualizes the process of perception and actualization of EHR affordances. The paper analyzes two-wave data from 91 healthcare professionals in an outpatient primary care clinic.

Paper 2 explores the influence of EHR adoption on the boundaries and practices of healthcare services delivery, as well as the consequences. Through a practice perspective, the paper analyzes the interplay between the field practices of individual communities and unifying boundary spanning practices.

Using 5 years of data from a Canadian EMR implementation, paper 3 proposes an affordance actualization process, which helps us to understand the changes that happen with new organizational IS. The paper finds that anchoring on legacy systems has a positive influence in affordance actualization.

Paper 4 investigates effective EMR use in a large acute care hospital. Using grounded theory, the paper examines five interrelated dimensions of consistency (process, meaning, form, place, content), and theoretical and practical implications of this enabler.

Paper 5 focuses on the user satisfaction aspect of health IS. The study found, by qualitative analysis, that 1) simpler systems are likely to be more satisfying than more complex systems, and 2) some system design and training considerations to consider.
Paper 6 found that designing caring and informative decision aids via the use of explanations and expressive speech acts can enhance the evaluation of the aids, thereby enhancing perceptions of its competence, benevolence, interaction atmosphere, user satisfaction, and overall interaction.

Paper 7, using the theory of workarounds, the IS success model, and Expectation Disconfirmation Theory, found that health IS users are motivated to work around the system if pre-adoption information quality expectations are unmet.

Paper 8 investigates the driving forces behind the adoption and use of a digital platform in a youth care organization. The study finds that capturing and sharing up-to-date expert knowledge in the USE IT-adoptionmodel is a positive factor in platform use.

In paper 9, an mHealth platform was built, tested, and found to facilitate evidence-based practice. The platform supports data collection, integration, reporting, and streamlining of workflows. Interviews and surveys were used to understand clinician perceptions and expectations.

Paper 10 suggests a framework to help explain the emergence of digitalization in healthcare. A longitudinal case study and interviews are used to interpret and contextualize the phenomenon, suggesting its enabling conditions, and intended / unintended outcomes.

Paper 11 reviews medical technology investment decision-making considerations. It finds that investment decisions lack comprehensive and data-driven investment decision methodologies. The study suggests developing a specific decision making process.

Paper 12 explores the possibilities of using drones as possible process optimization and cost saving tool. This new technology is evaluated through literature research and explorative expert interviews to identify the possible use and challenges.

Business-IT alignment concepts are reviewed and a novel method is proposed in the last paper. The findings in the Swiss hospital settings suggested that combination of IT management frameworks complimented by individual styles and behaviour considerations will lead to positive outcome. 